\title{
Journal of Clinical and Nursing Research
}

\author{
Research Article
}

\section{Study on Endoscopic Treatment of Non-Varicose Upper Gastrointestinal Bleeding}

Danyang $\mathrm{Li}^{1}$, Jianping $\mathrm{Hui}^{2^{*}}$

${ }^{1}$ Shaanxi University of Chinese Medicine First Clinical Medical School, Xianyang 712046, Shaanxi Province, China;

${ }^{2}$ Affiliated Hospital of Shaanxi College of Traditional Chinese Medicine, Xianyang 712046, Shaanxi Province, China

\begin{abstract}
Upper gastrointestinal bleeding is a common clinical disease with a relatively high incidence rate, and it is also an acute bleeding disease with a greater impact. If not rescued in time, it will greatly threaten the patient's physical and mental health and may even be fatal, especially in cases of non-varicose upper hemorrhage, which is closely related to digestive ulcers. In most cases, related diseases are self-limiting. However, some of the cases are relatively urgent and require internal meridian treatment to promote coagulation and improve existing disease situation. For endoscopic treatment process, related treatment methods have certain specificities, and their characteristics need to be fully understood.
\end{abstract}

Keywords: Endoscopic treatment; Non-varicose upper bleeding; Treatment method; Characteristics; Analysis

Publication date: November, 2020

Publication online: 30 November, 2020

*Corresponding author: Jianping Hui,

\section{Introduction}

1542579769@qq.com

Generally speaking, it is necessary to actively intervene during the treatment of upper hemorrhage according to its etiological characteristics. Most nonvaricose hemorrhages are mainly caused by factors such as mucosal rupture, and the amount of bleeding is relatively limited. But if the great vessels suffer invasion resulting in relatively large amount of bleeding, it may become fatal, hence requiring early targeted intervention. In terms of drug treatment, therapies such as acid suppression, coagulation and hemostasis are generally adopted, which are relatively poorly targeted, and it is difficult to effectively stop bleeding during the early stage of administration. In order to better adopt appropriate methods for treatment, endoscopic treatment should be selected based on the applicable symptoms to improve clinical treatment effectiveness. It is also necessary to fully understand the characteristics of such diseases to select relevant methods for treatment.

\section{Characteristics of non-varicose upper hemorrhage}

Clinical non-varicose bleeding is very common, which mainly refers to all bleeding phenomena caused by esophagus-gastric varicose except gastrointestinal bleeding. In cases of bleeding in the bile duct, gastrointestinal and esophagus, the incidence rate is relatively high and the disease mechanisms are relatively complex ${ }^{[1]}$. Compared with the upper blood loss caused by esophagus-gastric varicose, the risk of non-varicose bleeding is relatively lower, but its harmful effects should not be underestimated. The main causes of non-varicose bleeding include acute mucosal lesions, peptic ulcers, esophageal ulcers and esophagitis and gastric cancer etc. It displays typical clinical symptoms of hemorrhage, such as melena and hematemesis etc. Depending on the amount of bleeding, the severity of symptoms will also vary to a certain extent. It is worth mentioning that this type of disease can often lead to persistent bleeding in clinical practice, especially when the amount of bleeding is not large. The situation of persistent bleeding is relatively common. At the same time, if hemostasis is not complete, there is a possibility of recurrence. It 
is precisely because of this series of reasons that the mortality of related diseases is relatively high, and the quality of life of patients will be seriously affected. In current conventional treatment of the diseases, acid suppression and other treatments are generally adopted to improve the internal environment of the digestive tract and promote hemostasis. However, such methods have relatively limited effects. Some patients cannot effectively stop bleeding under conventional medical treatment.

\section{Endoscope Hemostasis and Precautions Needed}

Endoscopic hemostasis mainly uses electrocoagulation, local drug injection, vascular clipping, and local spraying of blood coagulation and vasoconstriction drugs to achieve rapid onset and precise treatment for further improving the patient's existing local bleeding problems ${ }^{[2]}$. In the specific treatment process, the injection of adrenaline can promote the contraction of local small arteries and veins, and effectively stop bleeding through compression. Therefore, this method can be used when the bleeding is not great and the small blood vessels are invaded. If bleeding of great vessels occurs, one can choose to use the method of clamping to operate, using mechanical force to close the local bleeding tissue and surrounding tissues, blocking the blood flow to further effectively stop the bleeding. However, it is worth mentioning that in the specific application process, the patient's current bleeding and ulcer conditions should be fully considered. If the scope is too large, it is not appropriate to adopt such a method, which may easily cause it to fall off, or incomplete clamping may occur. This means that in selecting specific process, the selection of methods such as local drug injection and physical hemostasis needs to be in line with the actual situation, especially the size of the bleeding surface and the existing situation. Certainly, other related methods have strong advantages with relatively good effects, and there are quite many specific hemostasis methods that can be selected. However, in practice, it is extremely necessary to carry out anti-shock and anti-anemia treatments promptly, and carry out related treatments when $\mathrm{Hb}$ returns to $70 \mathrm{~g} / \mathrm{L}$. If hemorrhagic shock is difficult to correct and active bleeding is relatively severe, anti-shock treatment can be performed during the process of endoscopic hemostasis, and the two can be performed in concert.
Since non-varicose upper hemorrhage has a certain possibility of recurrence, in the specific treatment process, there are also some independent factors that can easily lead to recurrence after endoscopic hemostasis. If the bleeding is rapid and the amount of bleeding is large, bleeding may occur again after effective hemostasis. Therefore, in the actual operation, one can choose physical and drug therapies to stop bleeding, and actively supplement coagulation factors to reduce the risk of rebleeding. In the case of a significant drop in hemoglobin level, it may also cause local blood supply and oxygen supply problems, and the healing of the lesion will be slowed down. Therefore, it is necessary to actively carry out basic treatment during the treatment process. Some special hemorrhages, such as bleeding from great vessels, can lead to jet-like hemorrhage. The pressure on the vascular stump is relatively high naturally, and failure to deal with it in time can easily lead to terminal bleeding due to pressure after hemostasis. In general, the hemostatic effect on patients is closely related to the size of the lesion, and the two are negatively correlated. The larger the lesion, the worse the hemostatic effect, and vice versa. In addition, it is necessary to strengthen nutritional support, administer proton pump inhibitors for acid suppression treatment, protect the gastric mucosa from recurring problems such as mucosal rupture, promote the improvement of local blood supply, and provide a good environment for wound healing.

\section{Conclusions}

In conclusion, the clinical advantages of endoscopic treatment for non-varicose hemorrhage are relatively obvious. In order to better perform follow-up operations during the learning process, we must first understand the applicable symptoms for endoscopic hemostasis and further clarify its advantages and required precautions.

\section{References}

[1] Hong YW. A clinical controlled study on the treatment of acute non-varicose upper gastrointestinal bleeding with metal titanium clips and injection hemostasis under endoscope $[\mathrm{J}]$. China Foreign Medical Treatment, 2018, 37(7): 79-80.

[2] Xin FY. Application value of endoscopic hemostasis in the treatment of acute non-varicose upper gastrointestinal bleeding[J]. Clinical Research and Practice, 2018, 3(11): 68-69. 